\title{
A Simple Scaling Analysis of X-ray Emission and Absorption in Hot-Star Winds
}

\author{
Stanley P. Owocki and David H. Cohen \\ Bartol Research Institute, University of Delaware, Newark, Delaware, 19716. E-mail: \\ owocki@bartol.udel.edu, cohen@bartol.udel.edu
}

\begin{abstract}
We present a simple analysis of X-ray emission and absorption for hot-star winds, designed to explore the natural scalings of the observed X-ray luminosity with wind and stellar properties. We show that an exospheric approximation, in which all of the emission above the optical depth unity radius escapes the wind, reproduces very well the formal solution for radiation transport through a spherically symmetric wind. Using this approximation we find that the X-ray luminosity $L_{\mathrm{X}}$ scales naturally with the wind density parameter $\dot{M} / v_{\infty}$, obtaining $L_{\mathrm{X}} \sim\left(\dot{M} / v_{\infty}\right)^{2}$ for optically thin winds, and $L_{\mathrm{X}} \sim\left(\dot{M} / v_{\infty}\right)^{1+s}$ for optically thick winds with an X-ray filling factor that varies in radius as $f \sim r^{s}$. These scalings with wind density contrast with the commonly inferred empirical scalings of X-ray luminosity $L_{\mathrm{X}}$ with bolometric luminosity $L_{\mathrm{Bol}}$. The empirically derived linear scaling of $L_{\mathrm{X}} \sim L_{\mathrm{Bol}}$ for thick winds can however be reproduced, through a delicate cancellation of emission and absorption, if one assumes modest radial fall-off in the X-ray filling factor $(s \approx-0.25$ or $s \approx-0.4$, depending on details of the secondary scaling of wind density with luminosity). We also explore the nature of the X-ray spectral energy distribution in the context of this model, and find that the spectrum is divided into a soft, optically thick part and a hard, optically thin part. Finally, we conclude that the energy-dependent emissivity must have a high-energy cut-off, corresponding to the maximum shock energy, in order to reproduce the general trends seen in X-ray spectral energy distributions of hot stars.
\end{abstract}

Subject headings: radiative transfer — shock waves — stars: early-type stars:mass-loss — X-rays: stars

\section{Introduction}

One of the great surprises of the Einstein X-ray satellite mission was the observation that hot, luminous, OB stars are strong emitters of soft $(\sim 1 \mathrm{keV})$ X-rays (Harnden et al. 1979; Seward et al. 1979). Unlike the cooler, late-type stars, for which the X-ray emission was found to scale with the star's rotation, the observed X-rays from hot stars show a roughly linear proportionality to the stellar bolometric luminosity, $L_{x} \approx 10^{-7} L_{b o l}$ (Long \& White 1980; Pallavacini et al. 1981; Chlebowski, Harnden, and Sciortino 1989). Observations from subsequent X-ray satellites, 
most notably ROSAT, have generally confirmed this earlier scaling result, with some minor refinements. For example, from a ROSAT survey of $42 \mathrm{O}$ stars, Kudritzki et al. (1996) find $\log L_{\mathrm{X}} / L_{\mathrm{Bol}} \approx-6.7 \pm 0.35$, with a somewhat tighter relationship when a weak dependence on a characterisic wind density $\dot{M} / v_{\infty}$ is included, $L_{\mathrm{X}} \propto\left(\dot{M} / v_{\infty}\right)^{-0.38} L_{\mathrm{Bol}}{ }^{1.34}$.

Even before these detections, X-ray emission from a narrow stellar "corona" was postulated to explain the superionization seen in the UV spectra of OB stars (Cassinelli \& Olson 1979). This coronal model was further refined by Waldron (1984), but other studies have limited the extent, temperature, and fractional contribution of the total X-ray output of such purported O-star coronae (Cassinelli, Olson, \& Stalio 1978; Nordsieck, Cassinelli, \& Anderson 1981; Cassinelli \& Swank 1983; Baade \& Lucy 1987; MacFarlane et al. 1993). A much more generally favored scenario has been that these X-rays arise from shocks that form in the wind from strong intrinsic instabilities in the line-driving mechanism (Lucy \& Solomon 1970; MacGregor, Hartmann, \& Raymond 1979; Owocki \& Rybicki 1984, 1985). Lucy \& White (1980) and Lucy (1982) proposed phenomenological models in which wind material accelerated by this line-driven instability rams into ambient, "shadowed" material, forming a forward shock that accelerates and heats that material, producing X-rays.

Dynamical simulation models of the nonlinear evolution of this instability have since indicated a quite different wind structure, dominated by reverse shocks that decelerate very rarefied, instability-accelerated material as it rams into compressed, dense shells ahead of it (Owocki, Castor, \& Rybicki 1988). Since only a small fraction of wind material is actually heated by passage through such reverse shocks, the resulting direct X-ray emission is very low, generally well below the observed value (Feldmeier et al. 1997a). To overcome this limitation, subsequent wind structure simulations by Feldmeier et al. (1997b) introduced a chaotic, turbulent perturbation at the photospheric lower boundary. This seeds a wind structure with substantial velocity dispersion among the dense shells, which thus collide amongst themselves, yielding much more shock-heated material. For the specific case of a massive OB supergiant wind with rather strong and chaotic base perturbations, the X-ray emission produced in such models can be made to approach the observed values. However, the dynamical simulations for particular cases have thus far made no attempt to explain the broad scaling of observed X-ray emission with stellar luminosity.

The goal of the present paper is to provide a firm basis for understanding these more general scaling properties of hot-star X-rays. Our approach here eschews detailed dynamical simulations, and instead examines phenomenalogical models of the X-ray emission, in order to define more clearly what overall properties are needed from instability-generated wind structure simulations to produce these observed scaling relations. A major point of our analysis is that a linear scaling of observed $L_{x}$ with $L_{b o l}$ does not arise naturally from expected properties of a wind-shock model, but requires a rather delicate balance of X-ray absorption and emission, which in turn requires a special form for the radial distribution of wind shocks. A further point is that such a balance between emission and absorption should be associated with specific trends in broad-band X-ray spectral properties. 
The remainder of this paper is organized as follows. Beginning from the formal solution to the radiation transfer equation for X-rays emitted throughout an expanding stellar wind (§2.1), we develop a simple "exospheric" approximation through which we derive a general scaling law for X-ray luminosity from a simple power-law emission model $(\S 2.2)$. By relating this to wind and stellar scaling laws we identify parameter requirements for reproducing the observationally inferred linear dependence of $L_{x}$ on $L_{b o l}(\S 2.3)$. We next examine the energy dependence of the $\mathrm{X}$-ray spectrum (§3.1) and then integrate the expression for the X-ray spectrum over energy in order to verify the result from the previous section that gave the linear $L_{\mathrm{X}} \sim L_{\text {Bol }}$ scaling (§3.2). The final section $(\S 4)$ summarizes our conclusions. In two appendices we examine the effect of assuming a constant wind velocity and verify the validity of the assumption of a smooth X-ray emissivity in terms of detailed numerical plasma emission models.

\section{Radiation Transfer Through an Expanding, Spherical Stellar Wind}

\subsection{Formal Solution for Constant Velocity Expansion}

To develop a simplified description of X-ray emission and absorption in a spherically symmetric, expanding envelope, we initially consider monochromatic X-rays, deferring the discussion of energy dependence to the next section. Let us first define the usual $(p, z)$ coordinates such that $z$ is a coordinate along a ray, and $p$ is the 'impact parameter', or minimum radial distance of the ray from the origin. From a formal solution of the transfer equation for X-rays emitted at a radius $r=\sqrt{p^{2}+z^{2}}$ with emissivity $\eta(r)$, the specific intensity observed at large radii $(z \rightarrow \infty)$ is

$$
I_{p}=\int_{-\infty}^{\infty} \eta(r) e^{-\tau(p, z)} d z
$$

The total X-ray luminosity is then given by integration over all rays

$$
L_{x}=8 \pi^{2} \int_{0}^{\infty} I_{p} p d p .
$$

Here the ray optical depth is of the form

$$
\tau(p, z)=\int_{z}^{\infty} \kappa_{x}\left(r\left(p, z^{\prime}\right)\right) \rho\left(r\left(p, z^{\prime}\right)\right) d z^{\prime}
$$

where $\rho$ is the mass density, and $\kappa_{x}$ is the X-ray absorption cross section per unit mass. We note that the opacity arises in relatively cool, unshocked portions of the wind, and is due primarily to K-shell bound-free transitions in $\mathrm{He}, \mathrm{C}, \mathrm{N}$, and $\mathrm{O}, \mathrm{Ne}, \mathrm{Na}, \mathrm{Mg}$, and $\mathrm{Si}$.

For an instability-generated shock model, X-ray emission is only expected once the wind has reached a substantial fraction $(\gtrsim 50 \%)$ of the terminal speed $v_{\infty}$, implying that the radial variation of mean wind density is within an order unity factor of that for a constant velocity (Owocki 1994; Feldmeier 1995). For simplicity, let us thus consider the case of expansion at a constant velocity 
$v_{\infty}$ (but see Appendix A for a treatment of a beta velocity law). Moreover, since ionization fractions are also fairly constant beyond about $0.5 v_{\infty}$ (MacFarlane, Cohen, \& Wang 1994), we assume also a radially constant X-ray mass-absorption coefficient, $\kappa_{x}$. The radial optical depth is then given simply by

$$
\tau(0, r)=\frac{\kappa_{x} \dot{M}}{4 \pi v_{\infty}} \int_{r}^{\infty} \frac{d r^{\prime}}{r^{\prime 2}}=\frac{R_{1}}{r}
$$

where $\dot{M}$ is the mass loss rate, and

$$
R_{1} \equiv \frac{\kappa_{x} \dot{M}}{4 \pi v_{\infty}}
$$

defines the radius of unit radial optical depth, i.e. $\tau\left(0, R_{1}\right) \equiv 1$. For nonradial $(p \neq 0)$ rays, we obtain

$$
\tau(p, z)=R_{1} \int_{z}^{\infty} \frac{d z^{\prime}}{p^{2}+z^{\prime 2}}=\frac{R_{1}}{p} \arccos \left[\frac{z}{\sqrt{p^{2}+z^{2}}}\right]=\frac{R_{1}}{p} \theta(p, z),
$$

where $\theta$ is the angle between the $+z$ ray direction and an outward radius vector. This suggests it would be convenient to recast the intensity integral (1) in terms of this angle,

$$
I_{p}=p \int_{0}^{\pi} \frac{\eta(r(p, \theta))}{\sin ^{2} \theta e^{R_{1} \theta / p}} d \theta .
$$

To proceed, we next note that X-ray emission arises primarily from two-body processes recombination and collisional excitation - and thus has a volume emissivity $\eta\left(\operatorname{ergs~s}^{-1} \mathrm{~cm}^{-3} \operatorname{ster}^{-1}\right)$ that is proportional to the square of the density,

$$
\begin{aligned}
\eta(r) & =\xi C_{s} f_{m} \rho^{2}(r) \\
& =\xi C_{s}^{2} f_{v} \rho^{2}(r),
\end{aligned}
$$

where $f_{m}$ is the mass fraction, or mass filling factor, of ambient wind that is X-ray emitting, and $C_{s} \equiv \rho_{s} / \rho$ is a factor to correct for any differences in the density $\rho_{s}$ of shocked material relative to the ambient wind density $\rho$ (which assumes a smooth wind, given by $\rho \equiv \dot{M} / 4 \pi r^{2} v$ ). We note that the factor $C_{s}$ accounts both for the post-shock compression and the deviations of the pre-shock density from the ambient wind density. The mass filling factor, $f_{m}$, is related to the volume filling factor by $f_{v}=f_{m} / C_{s}$. The emission coefficient $\xi\left(\operatorname{ergs~} \mathrm{cm}^{3} \mathrm{~s}^{-1} \mathrm{~g}^{-2}\right)$ is related to the commonly used cooling function $\Lambda$ (ergs s${ }^{-1} \mathrm{~cm}^{3}$ ) (e.g., Raymond \& Smith 1977) by $\xi=\Lambda / 4 \pi \mu_{e} \mu_{p}$, where $\mu_{e}$ and $\mu_{p}$ are the mean mass per electron and proton, respectively. Under typical stellar wind conditions $\xi$ is independent of density (at least when considered at the coarse spectral resolution associated with current X-ray detectors), and depends mainly on the electron temperature in the post-shock region. Assuming for now that the factor $\xi C_{s}^{2} f_{v}$ is spatially constant, the intensity integral (7) can be rewritten and evaluated as

$$
\begin{array}{r}
I_{p}=\frac{\xi C_{s}^{2} f_{v}}{p^{3}}\left(\frac{R_{1}}{\kappa_{x}}\right)^{2} \int_{0}^{\pi} \sin ^{2} \theta e^{-R_{1} \theta / p} d \theta \\
=2 \xi C_{s}^{2} f_{v} \frac{R_{1}}{\kappa_{x}^{2}}\left(\frac{1-e^{-\pi R_{1} / p}}{R_{1}^{2}+4 p^{2}}\right) .
\end{array}
$$


Application to the luminosity integral (2) then yields

$$
\begin{aligned}
L_{x}=4 \pi^{2} \xi C_{s}^{2} f_{v} \frac{R_{1}}{\kappa_{x}^{2}} \int_{0}^{\infty} \frac{1-e^{-2 \pi x}}{x\left(1+x^{2}\right)} d x \\
\quad \approx 9.76 \pi^{2} \xi C_{s}^{2} f_{v} \frac{R_{1}}{\kappa_{x}^{2}} \\
=2.44 \pi \frac{\xi C_{s}^{2} f_{v}}{\kappa_{x}} \frac{\dot{M}}{v_{\infty}}
\end{aligned}
$$

where the approximate equality follows from direct numerical evaluation of the integral. Note that, although the wind volume emission varies as the density squared, the X-ray luminosity escaping the wind scales only linearly with the density parameter $\dot{M} / v_{\infty}$, due to the effect of wind attenuation.

\subsection{Exospheric Approximation for Power-Law Radial Emission}

It is of interest to compare this numerical result with the heuristic formula from an "exospheric approximation" (Cohen et al. 1996) that estimates the X-ray luminosity from the volume integral of the outward (i.e. radiated into $2 \pi$ ster) emission beyond the radius with unit radial optical depth,

$$
L_{x} \approx 8 \pi^{2} \int_{R_{1}}^{\infty} \eta(r) r^{2} d r=\frac{2 \pi \xi C_{s}^{2} f_{v} \dot{M}}{\kappa_{x} v_{\infty}}
$$

where the latter equality follows from straightforward analytic integration. The rough agreement with the numerical result (eq. [10]) suggests that this simple exospheric formula should be quite useful for estimating $L_{x}$ in more complicated cases for which the required integrals are difficult to evaluate.

Let us thus next estimate $L_{x}$ for the somewhat more general case when the factor $\xi C_{s}^{2} f_{v}$ varies as a power-law beyond some lower boundary radius $R_{o}$ for X-ray emission,

$$
\begin{array}{rlrl}
\eta(r) & =\rho^{2}\left(\xi C_{s}^{2} f_{v}\right)_{o}\left(\frac{r}{R_{o}}\right)^{s} & & ; r>R_{o} \\
& =0 & ; r<R_{o}
\end{array}
$$

where $\left(\xi C_{s}^{2} f_{v}\right)_{o}$ is the emission factor at $r=R_{o}$. Unlike for the special case $R_{o}=R_{*}$ and $s=0$ considered above, exact evaluation for $L_{x}$ would now generally require numerical evaluation of a double integral over $p$ and $z$. However, application of the exospheric formula (eq. [11]) readily yields an approximate analytic scaling law for the X-ray luminosity,

$$
L_{x} \approx \frac{\left(\xi C_{s}^{2} f_{v}\right)_{o}}{2(1-s)}\left(\frac{\dot{M}}{v_{\infty}}\right)^{2} R_{o}{ }^{-s}\left(\max \left[R_{o}, R_{1}\right]\right)^{s-1} .
$$

We note parenthetically that this can be recast as a fitting formula,

$$
L_{x} \approx \frac{\left(\Lambda C_{s}^{2} f_{v}\right)_{o}}{2(1-s)} E M_{w}\left(\frac{R_{o}}{\max \left(R_{o}, R_{1}\right)}\right)^{1-s},
$$


where $E M_{w}=\int_{R_{o}}^{\infty} n^{2} d V o l$ is the total wind emission measure above $R_{o}$. Alternatively, we can express this in terms of the basic stellar wind parameters,

$$
\begin{aligned}
L_{x} & \approx \frac{\left(\xi C_{s}^{2} f_{v}\right)_{o}}{2(1-s) R_{o}}\left(\frac{4 \pi R_{o}}{\kappa_{x}}\right)^{1-s}\left(\frac{\dot{M}}{v_{\infty}}\right)^{1+s} & ; R_{o}<R_{1} \text { (optically thick) } \\
& \approx \frac{\left(\xi C_{s}^{2} f_{v}\right)_{o}}{2(1-s) R_{o}}\left(\frac{\dot{M}}{v_{\infty}}\right)^{2} & ; R_{o}>R_{1} \text { (optically thin). }
\end{aligned}
$$

Equations (15b) and (15b) represent the X-ray luminosity for, respectively, the 'thick' and 'thin' wind cases. Note that the luminosity now scales with $\left(\dot{M} / v_{\infty}\right)^{2}$ for the thin wind, but only with $\left(\dot{M} / v_{\infty}\right)^{1+s}$ for the thick wind.

We reiterate that these results have all been derived for monochromatic X-rays. We will see in $\S 3$ that a given stellar wind can be optically thick and some energies and optically thin at others, leading to spectral structure based on the energy-dependence of the X-ray attenuation.

\subsection{Relation to Wind Scaling Laws}

The above simple analysis illustrates that the most direct scaling of the X-ray luminosity should be with wind density, and not, as has generally been inferred empirically, with the bolometric luminosity. Let us thus next consider whether this inferred scaling could be explained through secondary scaling of wind density with luminosity. Within the standard Castor, Abbott,

and Klein (1975; hereafter CAK) theory for line-driven winds, the mass loss is predicted to scale as

$$
\dot{M} \sim L_{\mathrm{Bol}}^{1 / \alpha^{\prime}} M_{e f f}^{1-1 / \alpha^{\prime}}
$$

where $M_{e f f}$ is the effective stellar mass $\left(M_{e f f}=M_{*}\left(1-\Gamma_{E d d}\right)\right)$, and $\alpha^{\prime}=\alpha-\delta$ is a combination of the two line parameters, $\alpha$ and $\delta$ defined by CAK and Abbott (1982), which respectively describe the distribution of line opacity and its dependence on ionization level.

As first noted by Kudritzki, Lennon, and Puls (1995), this theoretical scaling is in good general accord with an empirically derived 'wind-momentum-lumosity' relation

$$
\dot{M} v_{\infty} R_{*}^{1 / 2} \sim L_{\mathrm{Bol}}^{1 / \alpha^{\prime}}
$$

wherein the connection with the CAK mass loss formula (16) follows from the scaling of the wind terminal speed with the surface escape speed,

$$
v_{\infty} \sim \sqrt{M_{e f f} / R_{*}}
$$

which is a well known theoretical (CAK; Pauldrach, Puls, \& Kudritzki 1986) and observational (e.g., Lamers, Snow, \& Lindholm 1995) result. For O supergiants, the extensive analysis by Puls 
et al. (1996) empirically obtained a wind-momentum-luminosity relation with a slope implying $\alpha^{\prime}=0.57$, which thus gives

$$
\frac{\dot{M}}{v_{\infty}} \sim \frac{L_{\mathrm{Bol}}{ }^{1.75} R_{*}^{0.5}}{M_{*}\left(1-\Gamma_{E d d}\right)} .
$$

The dependence on the Eddington factor is significant only for stars with very high luminosities. Between an early-B star like $\lambda$ Sco and an $\mathrm{O}$ supergiant like $\zeta$ Pup, $\left(1-\Gamma_{E d d}\right)$ changes by only a factor of 2 , while the bolometric luminosities vary by almost three orders of magnitude.

To convert eq. (19) to a scaling with luminosity alone, we can ignore the weak dependence on stellar radius; but we may want to allow for a systematic trend between luminosity and mass. For example, from stellar structure theory, one expects a quite strong trend e.g. $L_{\mathrm{Bol}} \sim M_{*}^{2.5}$, yielding $\dot{M} / v_{\infty} \sim L_{\mathrm{Bol}}{ }^{1.35}$. The observed $L_{\mathrm{X}} \sim L_{\mathrm{Bol}}$ relation would then be reproduced by the optically thick wind scaling relation $(15 \mathrm{~b})$ if $s \approx-0.25$.

By contrast, if we simply ignore any systematic trend between mass and luminosity, the observed $L_{\mathrm{X}} \sim L_{\mathrm{Bol}}$ relation would be reproduced by a filling-factor index $s \approx-0.40$. Either scaling is qualitatively consistent with detailed numerical simulations of the line-driven instability, which generally show a gradual drop-off in X-ray production with radius beyond the point where strong shocks begin to form in the wind. But overall it seems that, with such a wind-based model for X-ray emission, connection with the observed $L_{\mathrm{X}} \sim L_{\mathrm{Bol}}$ is inherently indirect, requring a rather specialized cancellation between the wind emission and absorption.

Finally, we note that for lower-density winds that are optically thin to X-rays, the X-ray luminosity becomes independent of $s$, scaling as

$$
L_{\mathrm{X}} \sim\left(\frac{\dot{M}}{v_{\infty}}\right)^{2} \sim L_{\mathrm{Bol}}^{2.7} \text { or } L_{\mathrm{Bol}}^{3.5},
$$

where the two scalings depend on whether one includes or ignores the systematic variation of luminosity with mass. Either form is in general accord with empirically inferred X-ray scalings, which show the dependence of X-rays on bolometric luminosity becomes much steeper around early-B stars, corresponding roughly to where the winds are becoming optically thin (Cohen et al. 1997).

\section{Energy Dependence}

\subsection{Power-law Absorption and Emission}

All of the above analysis is for monochromatic X-rays. However, current instruments (Einstein, ROSAT, ASCA) are sensitive to X-rays over almost two orders of magnitude in energy, and even with their quite modest spectral resolutions, have provided some basic information 
about the spectral energy distributions of X-rays in hot stars. So let us now consider the energy dependence of the absorption and emission, and of the resulting luminosity spectrum. Given the modest energy resolution of X-ray observations to date, we focus here on the broad-band spectral properties. The X-ray absorption - apart from several prominent K-shell edges - can be fit roughly by a power-law in energy,

$$
\kappa_{x}\left(E_{x}\right) \approx \frac{\kappa_{x o}}{E_{x}^{a}}
$$

Hillier et al. (1993) find, for $\zeta$ Pup (O4 If), $a \approx 2$ in layers where helium remains doubly ionized, and $a \approx 2.8$ where it has recombined to $\mathrm{He}^{+}$. For $\epsilon \mathrm{CMa}$ (B2 II), detailed calculations give $a \approx 1.8$ between $100 \mathrm{eV}$ and $1 \mathrm{keV}$ (Cohen et al. 1996). This energy dependence of opacity means a given wind can be optically thick at some energies, and optically thin at others. By setting $R_{1}=R_{o}$, we can derive a critical energy,

$$
E_{1}=\left(\frac{\kappa_{x o} \dot{M}}{4 \pi v_{\infty} R_{o}}\right)^{1 / a}
$$

that separates the parts of the spectrum for which the wind is optically thick $\left(E_{x}<E_{1}\right)$ from those for which the wind is optically thin $\left(E_{x}>E_{1}\right)$.

To derive the form of the luminosity spectrum one must specify the energy dependence of emission as well as the absorption. For simplicity let us again assume that the emissivity has a power-law energy dependence,

$$
\xi\left(E_{x}\right)=\frac{\xi_{*}}{E_{x}^{b}},
$$

which means that equation (12a) can be written as $\eta=\rho^{2}\left(\xi_{*} C_{s}^{2} f_{v}\right)_{o} E_{x}^{-b}\left(r / R_{o}\right)^{s}$. In Appendix B we show a comparison of this simple, smooth emissivity model to detailed numerical simulations of collisional equilibrium plasmas. This comparison shows that at the low resolution of current instruments, the line-dominated spectra are surprisingly well approximated by a smooth function such as equation (23), with a power-law spectrum being produced by a power-law temperature distribution. The luminosity spectrum is then given by,

$$
\begin{aligned}
\frac{d L_{x}}{d E_{x}} & =L_{1}\left(\frac{E_{x}}{E_{1}}\right)^{a(1-s)-b} ; E_{x}<E_{1} \\
& =L_{1}\left(\frac{E_{x}}{E_{1}}\right)^{-b} \quad ; E_{x}>E_{1}
\end{aligned}
$$

where the luminosity at the energy at which wind optical depth is unity, $L_{1} \equiv L_{x}\left(E_{1}\right)$, has the form

$$
L_{1}=\frac{\left(\xi_{*} C_{s}^{2} f_{v}\right)_{o}}{2 R_{o}(1-s)}\left(\frac{4 \pi R_{o}}{\kappa_{x o}}\right)^{b / a}\left(\frac{\dot{M}}{v_{\infty}}\right)^{2-b / a} .
$$

While equations (24a) and (24b) are convenient for plotting the spectrum for a given model, we can alternatively write this in a form that more explicitly displays the scalings with stellar 
parameters,

$$
\begin{aligned}
\frac{d L_{x}}{d E_{x}} & =\frac{\left(\xi_{*} C_{s}^{2} f_{v}\right)_{o}}{2 R_{o}(1-s)}\left(\frac{4 \pi R_{o}}{\kappa_{x o}}\right)^{1-s}\left(\frac{\dot{M}}{v_{\infty}}\right)^{1+s} E_{x}^{a(1-s)-b} & ; E_{x}<E_{1} \\
& =\frac{\left(\xi_{*} C_{s}^{2} f_{v}\right)_{o}}{2 R_{o}(1-s)}\left(\frac{\dot{M}}{v_{\infty}}\right)^{2} E_{x}^{-b} & ; E_{x}>E_{1}
\end{aligned}
$$

where, again, equations (26a) and (26b) represent the expressions for, respectively, the optically thick and optically thin wind domains (cf. eqs. 15b and 15b).

Using the simple power-law absorption and emission models, the energy-dependencies in the two domains have slopes that differ by $a(1-s)$. Furthermore, if $a(1-s)-b>0$ then $E_{x}=E_{1}$ is also the energy of peak flux, and the value of this peak is dictated solely by the absorption properties of the wind. Given equation (22) one would expect the observed values of $E_{1}$ to vary significantly from low-density B star winds to high-density O star winds. However, although some variation in the peak intensities are seen, it is much less dramatic than this model predicts. In addition, the relative slopes of the optically thin and optically thick parts of observed X-ray spectra are generally seen to differ by much more than $a(1-s)$, given reasonable values of $a$ and $s$. These trends are shown in Figure 1 for a representative $\mathrm{O}$ star ( $\zeta$ Pup) and a representative early-type B star ( $\lambda$ Sco).

It is thus apparent that a universal power-law emissivity function is too simple an assumption. An obvious problem with this functional description is that it implies the presence of plasma having an arbitrarily high temperature. It therefore seems appropriate to consider a truncation to the power-law emissivity introduced in equation (23) that corresponds roughly to the maximum shock energy in the wind. We therefore modify the emissivity as

$$
\xi\left(E_{x}\right)=\frac{\xi_{*}}{E_{x}^{b}} e^{-E_{x} / E_{\max }},
$$

where $E_{\max }$ is the maximum plasma temperature, corresponding to the maximum shock energy. The luminosity spectrum is now given by

$$
\begin{aligned}
\frac{d L_{x}}{d E_{x}} & =L_{1}\left(\frac{E_{x}}{E_{1}}\right)^{a(1-s)-b} e^{-E_{x} / E_{\max }} ; E_{x}<E_{1} \\
& =L_{1}\left(\frac{E_{x}}{E_{1}}\right)^{-b} e^{-E_{x} / E_{\max }} \quad ; E_{x}>E_{1}
\end{aligned}
$$

where $L_{1}$ has the same definition as before. The exponential cut-off in this model brings the luminosity spectrum more in line with the data shown in Figure 1, as well as that for most other OB stars.

With this general expression for the X-ray spectra, one sees that there are two different types of spectra that can occur. If $E_{\max }>E_{1}$ then the high-energy fall off of the spectrum occurs above $E_{\max }$, where the spectrum would be decreasing as a power-law already. Relative to the 


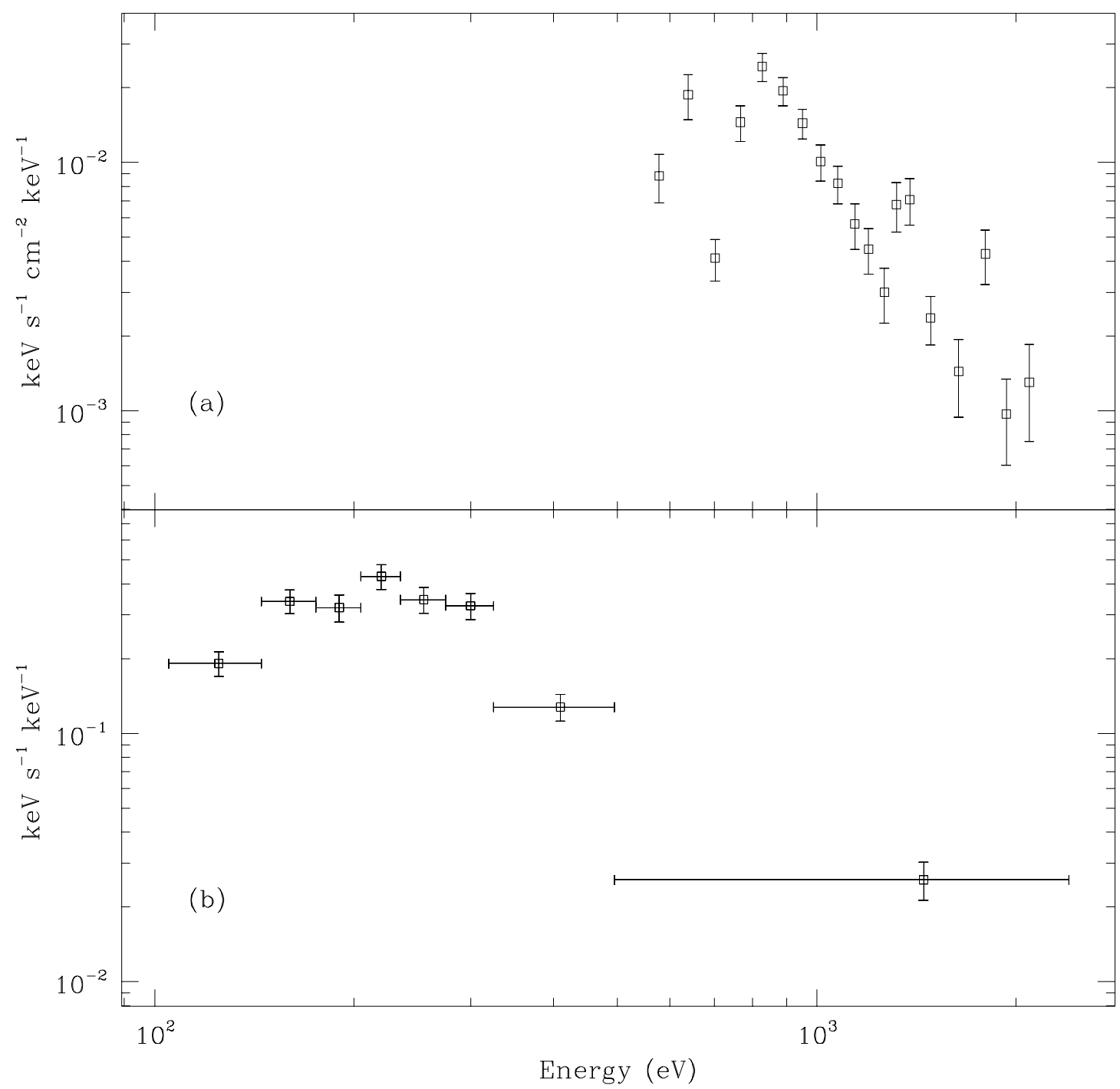

Fig. 1.- BBXRT (detector A) spectrum of $\zeta$ Pup (O4 If) taken from Corcoran et al. (1993) (a). This spectrum has been "unfolded" so as to correct for the energy-dependent sensitivity of the detector. Note the peak flux is near $0.7 \mathrm{keV}$, once the oxygen K-edge feature near this energy is corrected for. Given the parameters of $\zeta$ Pup $\left(\dot{M}=5 \times 10^{6} \mathrm{M}_{\odot}\right.$ yr ${ }^{-1} ; v_{\infty}=2.2 \times 10^{3} \mathrm{~km} \mathrm{~s}^{-1}$, $R_{*}=1.3 \times 10^{12} \mathrm{~cm}$ ) we find a value of $E_{1} \approx 2 \mathrm{keV}$, however. Note also that the power-law indices of the slopes of the low-energy and high-energy portions of the spectrum differ by at least six. The ROSAT PSPC spectrum of $\lambda$ Sco (B1.5 IV) (b) has a peak energy (corresponding to $E_{1}$ ) that is in much better agreement with the predicted value of $0.1 \mathrm{keV}$. The slightly higher observed peak value is entirely consistent with the finding that the mass-loss rates of $\mathrm{B}$ stars have been systematically underestimated (Cohen et al. 1997). 
simple power-law expression model, this spectrum has a peak determined by $E_{1}$. Effectively, then, the main change is to cause a much steeper high-energy fall-off in the spectrum. However, if $E_{\text {max }}<E_{1}$ then the peak in the spectrum is at a softer energy than it otherwise would have been (at an energy dictated by $E_{\max }$ rather than $E_{1}$ ), and the fall-off in the spectrum with increasing photon energy occurs that much sooner.

When O star X-ray data are fit with two-temperature models (e.g. Hillier et al. 1993), the higher temperature component tends to be approximately $5 \times 10^{6} \mathrm{~K}$, rarely exceeding $10^{7} \mathrm{~K}$. Even for the cooler B stars, the highest temperature component derived from model fitting is roughly $5 \times 10^{6} \mathrm{~K}$ (Cassinelli et al. 1994), indicating that $E_{\max }$ should be relatively constant for hot stars, with a value near $0.5 \mathrm{keV}$. This is in contrast to the values of $E_{1}$, which vary by more than an order of magnitude from mid-B to early-O stars. Therefore, which of these two cases applies to a given star depends primarily on the value of $E_{1}$, which is governed largely by the mass-loss rate. The case with $E_{\max }<E_{1}$ is thus appropriate to $\mathrm{O}$ stars, with their optically thick winds, while the case with $E_{\max }>E_{1}$ is relevant for B stars, with their thinner winds. For these low-density winds, the peak energy in the spectrum is determined by the value of $E_{1}$, whereas for the higher density, optically thick winds the peak of the spectrum is relatively constant at the universal value of $E_{\max } \approx 0.5 \mathrm{keV}$.

\subsection{The $L_{\mathrm{X}} / L_{\mathrm{Bol}}$ Scaling Relation from the Full Spectrum}

With the parameterization of the energy-dependence of wind attenuation and wind emission introduced in the previous subsection, we can return to the explanation for the scaling relationship discussed in $\oint 2.3$. Because the $\mathrm{O}$ stars and early-B stars for which the $L_{\mathrm{X}} / L_{\mathrm{Bol}}$ scaling holds have optically thick winds with $E_{\max }<E_{1}$, the optically thin portion of the wind $\left(E_{x}>E_{1}\right)$ contributes negligibly to the overall $\mathrm{X}$-ray luminosity and we therefore can consider only the optically thick expression, given by equation (28a). Furthermore, because of the negligible contribution of the high-energy portion of the spectrum, we can carry out the integration over all energies without significantly affecting the result. The total X-ray luminosity emergent from a hot star wind in this model is then given by

$$
\begin{aligned}
L_{x} \approx & \frac{\left(\xi_{*} C_{s}^{2} f_{v}\right)_{o}}{2 R_{o}^{s}(1-s)}\left(\frac{4 \pi R_{o}}{\kappa_{x o}}\right)^{1-s}\left(\frac{\dot{M}}{v_{\infty}}\right)^{1+s} \int_{0}^{\infty} E_{x}^{a(1-s)-b} e^{-E_{x} / E_{\max }} d E_{x} \\
& =\frac{\left(\xi_{*} C_{s}^{2} f_{v}\right)_{o}}{2 R_{o}^{s}(1-s)}\left(\frac{4 \pi R_{o}}{\kappa_{x o}}\right)^{1-s}\left(\frac{\dot{M}}{v_{\infty}}\right)^{1+s} E_{\max }^{1+a(1-s)-b} \Gamma(a-a s-b) .
\end{aligned}
$$

This expression preserves the $L_{\mathrm{X}} \sim\left(\dot{M} / v_{\infty}\right)^{1+s}$ dependence which yields $L_{\mathrm{X}} \sim L_{\mathrm{Bol}}$ with $s \approx-0.25$ or $s \approx-0.4$, as we derived in $\$ 2.3$ for monochromatic X-rays. We note that this analysis holds only for the optically thick winds that correspond to $\mathrm{O}$ stars which have $L_{\mathrm{X}} / L_{\mathrm{Bol}} \approx 10^{-7}$.

The relationship for monochromatic optically thin winds, $L_{\mathrm{X}} \sim\left(\dot{M} / v_{\infty}\right)^{2}$, is also consistent 
with the energy-dependent treatment, so long as $E_{1}$ is below the soft edge of the instrumental bandpass. Otherwise the observed spectrum has significant contributions from both an optically thin power-law region and an optically thick power-law region. In this hybrid case, the dependence of the X-ray luminosity on the bolometric luminosity is complicated and falls somewhere between the linear dependence of the thick winds and the much steeper dependence of the thin winds.

This would cause a less discontinuous fall-off in the $L_{\mathrm{X}} / L_{\mathrm{Bol}}$ relationship than is inferred from the monochromatic analysis in $\$ 2.3$.

\section{Conclusions}

Despite its approximate nature, the analysis described here provides a useful initial framework for examining the interplay among the basic processes of X-ray emission and absorption that determine the observed X-ray spectra from a hot-star wind.

Some of the main points are:

1. The simple procedure (cf. eq. [11]) of including only the X-ray emission above the radius $R_{1}$ of unit optical depth (the exospheric approximation) provides an accurate means to account for X-ray attenuation effects. A key aspect of this approximation is the assumption that $v \approx v_{\infty}$, which is indicated theoretically. If the $\mathrm{X}$-rays are formed deeper in the wind, then this constant-velocity approximation will underestimate the X-ray luminosity, but in Appendix A we estimate that the error is generally no more than about $50 \%$.

2. For optically thin winds, the X-ray luminosity increases as $\left(\dot{M} / v_{\infty}\right)^{2}$, as would be expected based on the density-squared sensitivity of the thermal emission processes. However, the dependence of the X-ray luminosity on wind density is less steep in the case of optically thick winds, with $L_{x} \sim\left(\dot{M} / v_{\infty}\right)^{1+s}$, where the X-ray filling factor has a power-law radial dependence, $f \sim r^{s}$.

3. Indeed, the natural scaling of the x-ray luminosity in hot-star winds is with the wind density parameter $\dot{M} / v_{\infty}$, and not with stellar parameters such as the bolometric luminosity.

4. However, if one introduces a radial power-law scaling of the filling factor, then the observed $L_{\mathrm{X}} \sim L_{\mathrm{Bol}}$ relation can be recovered with a value of $s \approx-0.25$ to $s \approx-0.4$ for the radial power-law index.

5. The energy dependence of the unit-optical-depth radius $R_{1}$ can play an important role in the shape of the energy spectrum. Indeed, in the simple shock model presented here, the peak of the spectrum occurs at the energy $E_{1}$ for which X-rays become optically thin at the initial shock onset radius $R_{o}$, as long as the emissivity does not begin to fall-off rapidly at energies below $E_{1}$. This condition should be satisfied for the low-density B star winds. However, for 
O star winds, the peak of the spectrum is governed by the high-energy cut-off in the shock strength distribution.

6. The X-rays observed by satellite telescopes are, especially for stars with optically thick winds, only a fraction of the total X-ray production. A significant amount of the X-ray production is absorbed by the optically thick winds of these stars. In addition, a significant amount of the total generated X-ray emission may fall outside of the observational bandpasses, especially in the very soft X-ray and EUV.

Future work will apply the principles illuminated here to interpretation of more fundamental, numerical simulations of X-ray emission from instability-generated shocks, to a detailed analysis of shock heating and cooling mechanisms, and to the interpretation of observational data. This will help clarify the strengths and weaknesses of the wind instability paradigm for explaining the observed X-ray spectra and scaling relations.

This research was supported in part by NASA grant NAG5-3530 to the Bartol Research Institute at the University of Delaware.

\section{A. Exospheric Model with a Beta Velocity Law}

Although there is good reason to believe that the X-ray production in hot star winds occurs primarily in regions of the wind where the velocity is close to the terminal velocity, it is possible to relax this assumption and still analytically solve for the X-ray luminosity under the exospheric approximation (eq. [11]), if the filling factor is radially constant.

The exospheric approximation is given by

$$
L_{x} \approx 8 \pi^{2} \int_{R_{1}}^{\infty} \eta(r) r^{2} d r
$$

where the emissivity $\eta \sim \rho^{2}$, and $R_{1}$ is the radius at which the radial optical depth is unity, $\tau\left(0, R_{1}\right) \equiv 1$. For a 'beta' velocity law of the form

$$
v(r)=v_{\infty}\left(1-R_{*} / r\right)^{\beta},
$$

the radial optical depth is given by the integral

$$
\tau(0, r)=\frac{\kappa_{x} \dot{M}}{4 \pi v_{\infty}} \int_{r}^{\infty} \frac{d r^{\prime}}{r^{\prime 2}\left(1-R_{*} / r^{\prime}\right)^{\beta}},
$$

which through a variable substitution $y=\left(1-R_{*} / r\right)$ can be readily evaluated as

$$
\tau(0, r)=\frac{\kappa_{x} \dot{M}}{4 \pi v_{\infty} R_{*}(1-\beta)}\left[1-\left(1-R_{*} / r\right)^{1-\beta}\right] .
$$


Setting $\tau=1$ leads to a new expression for the unit-optical-depth radius,

$$
R_{1}^{b e t a}=R_{*}\left[1-\left(1-\frac{R_{*}(1-\beta)}{R_{1}^{0}}\right)^{\frac{1}{1-\beta}}\right]^{-1}
$$

where $R_{1}^{0} \equiv \kappa_{x} \dot{M} / 4 \pi v_{\infty}$ is the previous result for a constant velocity flow, which simply represents the special case $\beta=0$.

Making the same change of variables in the exospheric expression for the X-ray luminosity and using the newly defined lower limit of integration, $R_{1}^{b e t a}$, one finds

$$
L_{x}^{\text {beta }}=\frac{\xi C_{s}^{2} f_{v} \dot{M}^{2}}{2 R_{*}(1-2 \beta) v_{\infty}^{2}}\left[1-\left(1-\frac{R_{*}(1-\beta)}{R_{1}^{0}}\right)^{\frac{1-2 \beta}{1-\beta}}\right] .
$$

Scaled by the constant-velocity exospheric expression (eq. 11]), this can be cast as a correction factor,

$$
L_{x}^{\text {beta }} / L_{\mathrm{X}}=\frac{R_{1}^{0}}{R_{*}(1-2 \beta)}\left[1-\left(1-\frac{R_{*}(1-\beta)}{R_{1}^{0}}\right)^{\frac{1-2 \beta}{1-\beta}}\right]
$$

Note that as $R_{1}^{0} / R_{*}$ approaches infinity, $L_{x}^{\text {beta }} / L_{\mathrm{X}}$ approaches unity. Furthermore, for $\beta=0.8$ if $R_{1}=2 R_{*}$ then $L_{x}^{\text {beta }} / L_{\mathrm{X}} \approx 1.25$. Choosing an $R_{1}^{0}$ that gives $R_{1}^{\text {beta }}=1.5 R_{*}$, which seems a reasonable minimum radius for onset of instability-generated X-rays, $L_{x}^{\text {beta }}$ is just over $50 \%$ greater than $L_{\mathrm{X}}$.

\section{B. Comparison of Power-law Emission Spectra to Numerical Models}

Given that the optically thin, thermal emission expected from the shock-heated regions of hot-star winds is dominated by lines (e.g. Raymond \& Smith 1977; Mewe, Groenenschild, \& van den Oord 1985), we may ask how well the smooth power-law models we have assumed in this paper represent the true spectra. We thus created a series of collisional equilibrium spectral models using the MeKaL code (Mewe, Kaastra, Liedahl 1995) and assuming B-star abundances (Geis \& Lambert 1992). These are power-law differential emission models of the form $\frac{d E M}{d T} \sim 1 / T^{c}$ that are constructed from a superposition of multiple isothermal models. The individual isothermal models used to make the power-law differential emission models have temperatures on the interval $5.0 \leq \log T(\mathrm{~K}) \leq 7.7$. The spectra were calculated on the range $15 \mathrm{eV}$ to $2500 \mathrm{eV}$. Although 100 $\mathrm{eV}$ is generally taken to be the lower bound of the X-ray bandpass, significant emission occurs at lower energies, even for very hot plasma. We have compared these single power-law temperature distribution models to the single power-law spectral models described by equation (23).

Currently, the best spectral resolution available from space-based observatories is $\lambda / \Delta \lambda \approx 20$ from $B B X R T$ and $A S C A$. We therefore binned the detailed models at slightly better than this 
resolution, and fit the resulting spectra with single power-laws, $\xi \sim E_{x}^{-b}$. Three examples of this exercise are shown in Figure 2. The power-law assumption is seen to be quite good. Reasonable $\chi^{2}$ values result from these fits if statistical uncertainties of only a factor of 2 to 3 are assumed for each bin. It should be kept in mind that the actual resolution of most available X-ray data sets is significantly worse than the binning of these models indicate, so that the observed data are, in fact, even more well-described by power-laws.

There are several interesting things to note from this exercise. First, the global structure in these model spectra are dominated by the emission bump centered near $0.8 \mathrm{keV}$. This feature is due to thousands of iron L-shell emission lines. Second, a very significant fraction of the flux emerges not in the soft X-ray, but actually in the EUV, below $100 \mathrm{eV}$. Although this radiation is very significant (not least because of the role it plays in determining the wind excitation and ionization conditions), it has been directly observed in only one hot star, $\epsilon$ CMa (Cohen et al. 1996). Finally, the best-fit spectral power-law slopes are generally steeper than the power-law slopes of the differential emission measure (plasma temperature) distributions by roughly 0.7. This factor is a reflection of the fact that on the temperature range $10^{5}<\mathrm{T}<10^{7}$, hotter plasma emits less efficiently than cooler plasma. Indeed, the slope of the frequency-integrated cooling-curve between the temperatures of $10^{5}$ and $10^{7} \mathrm{~K}$ (e.g. Raymond, Cox, \& Smith 1976) is very close to the value 0.7 . 


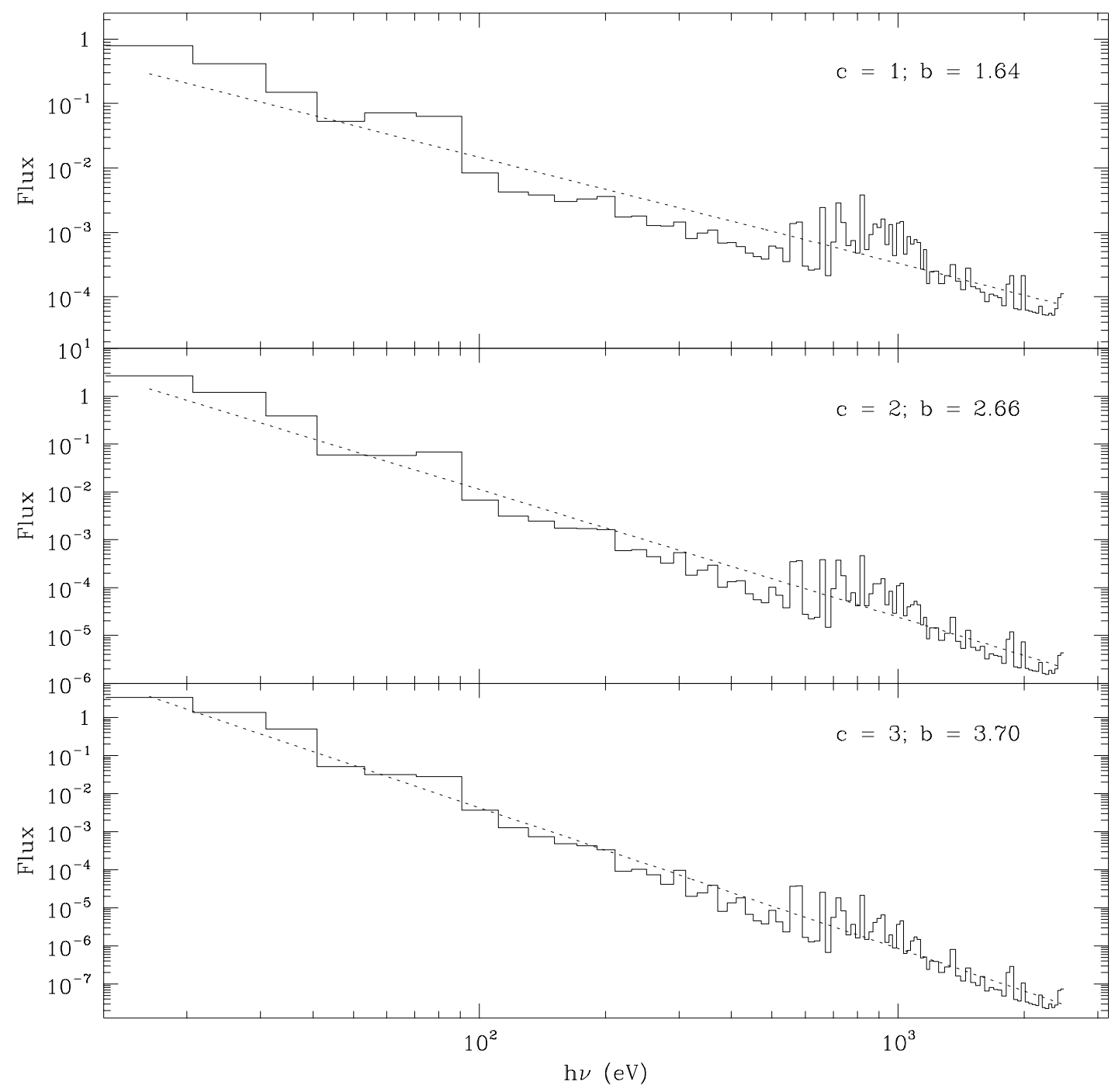

Fig. 2.- Temperature power-law spectral models (solid lines) calculated from the MeKaL code are shown along with the power-law spectral fits (dotted lines). The best-fit power-law spectral indices (b) are indicated along with the differential emission measure slope used in making the models (c). 


\section{REFERENCES}

Abbott, D. A. 1982, ApJ, 263, 723

Baade, D., \& Lucy, L. B. 1987, A\&A, 178, 213

Cassinelli, J. P., Cohen, D. H., MacFarlane, J. J., Sanders, W. T., \& Welsh, B. Y. 1994, ApJ, 421, 705

Cassinelli, J. P., \& Olson, G. L. 1979, ApJ, 229, 304

Cassinelli, J. P., Olson, G. L., \& Stalio, R. 1978, ApJ, 220, 573

Cassinelli, J. P., \& Swank, J. H. 1983, ApJ, 271, 681

Castor, J. I., Abbott, D. C., \& Klein, R. I. 1975, ApJ, 195, 157 (CAK)

Chlebowski, T., Harnden, F. R., Jr., \& Sciortino, S. 1989, ApJ, 341, 427

Cohen, D. H., Cassinelli, J. P., \& MacFarlane, J. J. 1997, ApJ, 487, 867

Cohen, D. H., Cooper, R. G., MacFarlane, J. J., Owocki, S. P., Cassinelli, J. P., \& Wang, P. 1996, ApJ, 460, 506

Corcoran, M. F., et al. 1993, ApJ, 412, 792

Feldmeier, A. 1995, A\&A, 299, 523

Feldmeier, A., Kudritzki, R.-P., Palsa, R., Pauldrach, A. W. A., \& Puls, J. 1997a, A\&A, 320, 899

Feldmeier, A., Puls, J., \& Pauldrach, A. W. A. 1997b, A\&A, 322, 878

Geis, D. R., \& Lambert, D. L. 1992, ApJ, 387, 673

Harnden, F. R., Jr., Branduardi, G., Gorenstein, P., Grindlay, J., Rosner, R., Topka, K., Elvis, M., Pye, J. P., \& Vaiana, G. S. 1979, ApJ, 234, L51

Hillier, D. J., Kudritzki, R. P., Pauldrach, A. W. A., Baade, D., Cassinelli, J. P., Puls, J., \& Schmitt, J. H. M. M. 1993, A\&A, 276, 117

Kudritzki, R. P., Lennon, D., and Puls, J. 1995, in Science with the Very Large Telescope, eds. J. Walsh and I. Danziger, (Springer: Heidelberg), 246

Kudritzki, R. P., Palsa, R., Feldmeier, A., Puls, J., \& Pauldrach, A. W. A. 1996, Röntgenstrahlung from the Universe, ed. H. U. Zimmermann, J. Trümper, \& H. York (MPE: Garching), 9

Lamers, H. J. G. L. M., Snow, T. P., \& Lindholm, D. M. 1995, ApJ, 455, 269

Long, K. S., \& White, R. L. 1980, ApJ, 239, L65

Lucy, L. B., \& Solomon, P. M. 1970, ApJ, 159, 879

Lucy, L. B. 1982, ApJ, 255, 286

Lucy, L. B., \& White, R. L. 1980, ApJ, 241, 300

MacFarlane, J. J., Waldron, W. L., Corcoran, M. F., Wolff, M. J., \& Wang, P. 1993, ApJ, 419, 813

MacFarlane, J. J., Cohen, D. H., \& Wang, P. 1994, ApJ, 437, 351 
MacGregor, K. B., Hartmann, L., \& Raymond, J. C. 1979, ApJ, 231, 514

Mewe, R., Gronenschild, E. H. B. M., \& van den Oord, G. H. J. 1985, A\&AS, 62, 197

Mewe, R., Kaastra, J. S., \& Liedahl, D. A. 1995, Legacy, issue 6, 16

Nordsieck, K. H., Cassinelli, J. P., \& Anderson, C. M. 1981, ApJ, 248, 678

Owocki, S. P. 1994, Ap\&SS, 221, 30

Owocki, S. P., Castor, J. I., \& Rybicki, G. B. 1988, ApJ, 335, 914

Owocki, S. P., \& Rybicki, G. B. 1984, ApJ, 284, 337

Owocki, S. P., \& Rybicki, G. B. 1985, ApJ, 299, 265

Pallavicini, R., Golub, L., Rosner, R., Vaiana, G. S., Ayres, T., \& Linsky, J. L. 1981, ApJ, 248, 279

Pauldrach, A. W. A., Puls, J., \& Kudritzki, R.-P. 1996, A\&A, 164, 86

Puls, J., Kudritzki, R.-P., Herrero, A., Pauldrach, A. W. A., Haser, S. M., Lennon, D. J., Gabler, R., Voels, S. A., Vilchez, J. M., Wachter, S., \& Feldmeier, A. 1996, A\&A, 305, 171

Raymond, J. C., Cox, D. P., \& Smith, B. W. 1976, ApJ, 204, 290

Raymond, J. C., \& Smith, B. W. 1976, ApJS, 35, 419

Seward, F. D., Forman, W. R., Giacconi, R., Griffiths, R. E., Harnden, F. R., Jr., Jones, C., \& Pye, J. P. 1979, ApJ, 234, L55

Waldron, W. L. 1984, ApJ, 282, 256 